\title{
NGS barcode sequencing in taxonomy and diagnostics, an application in "Candida" pathogenic yeasts with a metagenomic perspective
}

\author{
Claudia Colabella ${ }^{1}$, Laura Corte ${ }^{1}$, Luca Roscini ${ }^{1}$, Matteo Bassetti ${ }^{2}$, Carlo Tascini ${ }^{3}$, Joseph C. Mellor ${ }^{4}$, Wieland Meyer ${ }^{5}$, Vincent \\ Robert $^{6}$, Duong $\mathrm{Vu}^{6}$, and Gianluigi Cardinali ${ }^{1,7}$
}

\begin{abstract}
${ }^{1}$ Microbiology Section, Department of Pharmaceutical Sciences, University of Perugia, 06121, Italy
${ }^{2}$ Infectious Diseases Division, Santa Maria Misericordia University Hospital, Udine, 33100, Italy

${ }^{3}$ Infectious Diseases Division, Cotugno Hospital Napoli, 80131, Italy

${ }^{4}$ seqWell Inc., 376 Hale Street, Beverly, MA 01915, USA Australia

${ }^{6}$ Bioinformatics Unit, Westerdijk Fungal Biodiversity Institute, $3508 \mathrm{CT}$, Utrecht, Netherlands cardinali@unipg.it

Abstract: Species identification of yeasts and other Fungi is currently carried out with Sanger sequences of selected molecular markers, mainly from the ribosomal DNA operon, characterized by hundreds of tandem repeats of the 18S, ITS1, 5.8S, ITS2 and LSU loci. The ITS region has been recently proposed as a primary barcode marker making this region the most used one in taxonomy, phylogeny and diagnostics. The introduction of NGS is providing tools of high efficacy and relatively low cost to amplify two or more markers simultaneously with great sequencing depth. However, the presence of intra-genomic variability between the repeats requires specific analytical procedures and pipelines. In this study, 286 strains belonging to 11 pathogenic yeasts species were analysed with NGS of the region spanning from ITS1 to the D1/D2 domain of the LSU encoding ribosomal DNA. Results showed that relatively high heterogeneity can hamper the use of these sequences for the identification of single strains and even more of complex microbial mixtures. These observations point out that the metagenomics studies could be affected by species inflection at levels higher than currently expected.
\end{abstract}

${ }^{5}$ Molecular Mycology Research Laboratory, Centre for Infectious Diseases and Microbiology, Sydney Medical School, Westmead Hospital, Marie Bashir Institute for Infectious Diseases and Biosecurity, The University of Sydney, Westmead Institute for Medical Research, Sydney, NSW 2006,

${ }^{7}$ CEMIN Research Centre of Excellence, University of Perugia, Borgo 20 Giugno 74, 06121, Italy; corresponding author e-mail: gianluigi.

\author{
Key words: \\ identification \\ ITS
}

LSU

Next generation sequencing Sanger

Article info: Submitted: 21 July 2017; Accepted: 10 May 2018; Published: 22 May 2018.

\section{INTRODUCTION}

The regions encoding for the ribosomal DNA in yeasts are organized in an array ranging from 10 to $20 \mathrm{~kb}$ according to the species, including the small subunit (18S), the internal transcribed spacers (ITS1 and ITS2) and the large subunit (5S, 5.8S and 26S) (Dujon et al. 2004). These arrays vary in number from a few dozen to hundreds (Maleszka \& Clark-Walker 1993, Amend et al. 2010). In Candida albicans, the operon is $12756 \mathrm{bp}$ long and the diploid genomes can contains 110 repeats in a single locus (Jones et al. 2004), whereas in Candida glabrata these sequences are dispersed in two subtelomeric regions (Maleszka \& Clark-Walker 1993, Dujon et al. 2004). This difference reflects the taxonomic distance $(C$. albicans belongs to Debaromycetacee whereas C. glabrata belongs to Saccharomycetaceae) and the evolution, the former being a pre-genome-duplication species and the latter a postgenome-duplication species (Dujon 1996). The sequences of these genes have been largely used in the last decades in taxonomy and phylogenetic studies thanks to their high conservation (Kurtzman \& Robnett 1998, Groenewald et al. 2011), by means of Sanger sequencing that produces a single sequence of each gene. However, secondary peaks have been observed suggesting some level of heterogeneity among the various copies of the tandem repeats in Fungi (Korabecna 2007, Woo et al. 2010, Vydryakova et al. 2012, Li et al. 2014), yeasts (Alper et al. 2011), ciliates (Gong et al. 2013) and in some plants (Wang et al. 2015). The extent of this variability is critical for the exact understanding governing the homogenization of multigene families, which is typically attributed to concerted evolution, i.e. the internal evolution of the members of a multigene family that mutate over the time more or less in the same way. An effect is that the paralogous genes within a species are much closer to one another than to the same genes in other species. This phenomenon can be explained by gene conversion or asymmetric crossing-over (Liao 1999, Nei \& Rooney 2005,

Cㅇ 2018 International Mycological Association

You are free to share - to copy, distribute and transmit the work, under the following conditions:

Attribution: $\quad$ You must attribute the work in the manner specified by the author or licensor (but not in any way that suggests that they endorse you or your use of the work).

Non-commercial: $\quad$ You may not use this work for commercial purposes.

No derivative works: You may not alter, transform, or build upon this work.

For any reuse or distribution, you must make clear to others the license terms of this work, which can be found at http://creativecommons.org/licenses/by-nc-nd/3.0/legalcode. Any of the above conditions can be waived if you get permission from the copyright holder. Nothing in this license impairs or restricts the author's moral rights. 
Ganley \& Kobayashi 2007, Naidoo et al. 2013). Another model to explain the copy homogenization is the birth-anddeath model, according which new versions of the genes appear with the "birth" transition and all the variants, but one (or very few) are purged during the "death" transition (Nei \& Rooney 2005). However, the possibility of explaining this homogenization of the rDNA loci with the birth-and-death model is still a matter of debate, and some authors have claimed that the variation observed fits this model better than that of concerted evolution (Nei \& Rooney 2005). In general, it is possible that different mechanisms have been active in different taxa, and maybe even in different regions (Vydryakova et al. 2012). A mixed model of evolution involving both models simultaneously was considered, although not for this gene family (Nei \& Rooney 2005). The major differences between these models are that concerted evolution is expected to produce scarce heterogeneity, whereas the birth-and-death mechanism should yield more Intra-Genomic Polymorphisms (IGP) (Ganley \& Kobayashi 2007). From the above observations it appears that concerted evolution is not a satisfactory model when IGPs frequency is particularly high (Simon \& Weiß 2008).

Beyond the model governing the homogenization of the repeat units, the internal variability within the rDNA is a source of additional information useful in phylogenetic, environmental, and clinical microbiology, to trace the origin of strains (West et al. 2014). As long as Sanger sequencing was the sole or predominant technology, the sequence reported the most frequent nucleotides hiding the least frequent, occasionally visible as secondary peaks (Woo et al. 2010).

Since ITS has been agreed as the universal barcode for Fungi (Schoch et al. 2012), the possibility of applying Next Generation Sequencing (NGS) to this locus offers several advantages, such as the possibility of studying microbial communities, independently of their viability and capacity of growing on existing media (Bokulich \& Mills 2012, Hajibabaei 2012). Problems still exist in the exact quantification of taxa on the basis of NGS read abundance (Amend et al. 2010), and care should be taken in data analysis because the internal heterogeneity could cause an inflation of the species richness (Lindner \& Banik 2011, Lindner et al. 2013). Since database quality and completeness are mandatory for NGS analyses (Bokulich \& Mills 2012), the presence of a few alternative barcode markers proposed and under evaluation (Stielow et al. 2015) leads to the use of the rDNA genes for identification, although a much deeper understanding of the problems and effective analytical pipelines are necessary (Medinger et al. 2010).

NGS technology is now sufficiently mature to move from specialized research centres to environmental and clinical laboratories. However, the massive amount of data and the internal heterogeneity can be a serious problem to get sound and rapid high-throughput identifications (Ahmed 2016), especially when a more complex metagenomics approach is taken to describe microbial communities in patients and healthy controls (Imabayashi et al. 2016). Among the various approaches described, a BLAST search followed by an assembly that is further tested with BLAST has been recently described and proposed (Ahmed 2016, Imabayashi et al. 2016).
In this paper we describe an innovative system of yeast strain identification using next generation sequencing of amplicons covering the region spanning the ITS1 to the D1/ D2 domain of the LSU. The former region is that accepted as the universal barcode in Fungi (Schoch et al. 2012) and is now included in highly curated databases (Schoch et al. 2014, Irinyi et al. 2015); the latter was the first locus introduced in yeast identification (Kurtzman \& Robnett 2013, Susca et al. 2013, Yurkov et al. 2015). These regions were amplified simultaneously in single amplicons in order test two hypotheses: (1) the possibilities offered by NGS for amplicon based single strain identification; and (2) the accuracy of this technique in a metagenomic study. The latter concept is based on the multi-copy nature of these markers, and their intrinsic endogenous variability, which could lead to the erroneous attribution of the reads to the right species and also to other ones. With our settings, it is possible to evaluate the rate of erroneous identifications caused by the above issues.

The analysis was performed with 286 strains of pathogenic yeasts isolated from two Italian hospitals, which had previously been studied to show that the success of these strains in the hospital environment is strictly related to their ability to form biofilms (Corte et al. 2016). This set of strains is large enough to represent the identification routine occurring in a clinical setting, as well as in other environmental studies, and to pave the way to further studies on the composition of repeats present in the rDNA region.

\section{MATERIALS AND METHODS}

\section{Strains and growth conditions}

The 286 strains of opportunistic Candida species analysed (Table S1) were isolated from patient blood cultures obtained in two Italian hospitals in Pisa and Udine; these had been incorporated into the Cemin Microbial Collection of the Microbial Genetics and Phylogenesis Laboratory (Cemin, Centre of Excellence on Nanostructured Innovative Materials for Chemicals, Physical and Biomedical Applications, University of Perugia). Candida albicans, C. parapsilosis, C. tropicalis, and C. glabrata represented the majority of the isolates. The first three species are phylogenetically related among themselves, where $C$. glabrata is more closely related to $S$. cerevisiae. Strains were stored at $-80{ }^{\circ} \mathrm{C}$ in $17 \%$ glycerol immediately after isolation. Short-term storage was carried out on YEPDA (YEPD supplemented with $1.7 \%$ agarose) at $4^{\circ} \mathrm{C}$. Strains were grown in YEPD (yeast extract $1 \%$, peptone $1 \%$, and dextrose $1 \%$ ); all products were obtained from Biolife (http://www.biolifeitaliana.it/) and kept at $37^{\circ} \mathrm{C}$ with $150 \mathrm{rpm}$ shaking.

\section{DNA extraction and molecular techniques}

Genomic DNA was extracted as indicated by Cardinali et al. (2001). The ITS1, 5.8S, ITS2 rDNA gene cluster regions and the D1/D2 domain of the LSU gene were amplified with FIREPole ${ }^{\circledR}$ Taq DNA Polymerase (Solis BioDyne, Estonia), using the ITS1 (5'-TCCGTAGGTGAACCTGCGG) - NL4 (GGTCCGTGTTTCAAGACGG) primers pair. The amplification protocol was carried out as follows: initial 
denaturation at $94{ }^{\circ} \mathrm{C}$ for $3 \mathrm{~min}, 30$ amplification cycles (94 ${ }^{\circ} \mathrm{C}$ for $1 \mathrm{~min}, 54{ }^{\circ} \mathrm{C}$ for $1 \mathrm{~min}$ and $72{ }^{\circ} \mathrm{C}$ for $1 \mathrm{~min}$ ) and final extension at $72{ }^{\circ} \mathrm{C}$ for $5 \mathrm{~min}$. Amplicons were subjected to electrophoresis a $1.5 \%$ agarose gel (Gellyphor, EuroClone, Italy). Amplicons were sequenced with NGS plexWell ${ }^{\text {TM }}$ technologies (http://www.seqwell.com/) with the same primers used for the generation of the amplicons. The reads of each strain, contained in FASTQ file, were analysed with Geneious R9 (Kearse et al. 2012).

\section{Bioinformatics analysis}

Mapping against a reference vs de novo assembling

Mapping and assembling analyses were carried out on 12 strains (Table 1), representative of six species showing different numbers of reads, ranging from 19388 to 53497 . Each strain was mapped against the ITS-LSU concatenate rDNA sequences of the respective ex-type strain, used as reference and provided by the fungal collection database of the Westerdijk Fungal Biodiversity Institute (CBS): CBS 562 C. albicans, CBS 138 C. glabrata, CBS 604 C. parapsilosis, CBS 10906 C. orthopsilosis, CBS 94 C. tropicalis, and CBS 2030 Meyerozyma guilliermondii.

The 12 FASTQ files were filtered to remove reads shorter than $140 \mathrm{bp}$. For the mapping analysis, contigs were obtained using two algorithms: Bowtie2 with setting "local" (hereinafter referred to as BTL) searching only for the best match (Langmead \& Salzberg 2012) and BBMap $(\mathrm{BBm})$ set to mapping as multiple best matching in random mode (Bushnell 2014). The two algorithms can align reads from all major NGS platforms; BTL is typically used with short reads and large reference sequences while $\mathrm{BBm}$ is particularly used with highly mutated sequences or reads with long indels; a comparison between the two algorithms has been reported (Berrocal et al. 2016, Lubock et al. 2017). Mappings were performed using High Sensitivity mode and no trimming of the sequences. De novo assembling was performed using Geneious assembler after testing other algorithms, with High and Low sensitivity settings without trimming. Contig identification was carried out with BLAST search using a local library containing 15 ITSLSU concatenate rDNA sequences of the Candida ex-type strains (Table 2).

The Saccharomyces cerevisiae CBS 1171 ex-type strain was used as outgroup. The highest similarity matches were searched for using Megablast.

\section{Mapping against a reference using large libraries}

Mapping procedures were performed using strains CMC 1793 and CMC 1818 with 21238 and 58263 reads respectively and three selected and accurate libraries: Westerdijk Fungal Biodiversity Institute collection database (CBS) containing ITS sequences and ITS-LSU sequences of ex-type strains, and the ISHAM-ITS database (http://its. mycologylab.org/) containing ITS sequences of medical related strains. The two FASTQ files were mapped against the different libraries using the two algorithms BTL setting "local" and BBm with High Sensitivity mode and no trimming of the sequences.

\section{Mapping against a selected library (M1)}

All the 286 FASTQ files were filtered to remove reads shorter than $140 \mathrm{bp}$ and were mapped against the local library of 16 ITS-LSU concatenate rDNA sequences of ex-type strains using two algorithms (BTL and BBm) with High Sensitivity mode and no trimming. All the 16 ITS-LSU rDNA sequences were provided by the fungal collection database of the Westerdijk Fungal Biodiversity Institute (CBS). Results were exported from Geneious R9 software in Microsoft Excel ${ }^{\circledR}$. Using a built-in macro, six indices were calculated for both algorithms and data were assembled in order to give an unambiguous taxonomic interpretation of the results.

- Iread: This index is the ratio of the number of reads attributed to each member of the reference library, and therefore indicates the share of reads $\left(R_{i}\right)$ attributable to the species represented by the ex-type strain $(R)$.

- Inuc: This index is the ratio of the nucleotides mapped with the ex-type strain $\left(N_{i}\right)$ on the total number of nucleotides present on the reads of the FASTQ file (N).

- Icov: This index of coverage describes the average number of reads that align to, or "cover", known reference bases. At higher levels of coverage, each base is covered by a greater number of aligned sequence reads, so base calls can be made with increased confidence. The index is represented by the ratio of coverage value of the strain $\left(C_{i}\right)$ on the total coverage of the reads $(C)$.

- Iref: Refseq percentage.

- Isim: Similarity percentage.

- Isyn: This index represents the ratio of the sum of all the five indices of one species (Isp) to the sum of the indices of all the species (Itot).

Mapping against the ex-type strain of the presumptive species and "unused reads" (M2 followed by the final M3 mapping)

All the 286 FASTQ files were mapped against the ITS-LSU concatenated sequences of the ex-type strains resulting from the first mapping (M1). The second mapping procedure was performed using the two algorithms (BTL and BBm) with High Sensitivity mode and no trimming. Results were exported from Geneious R9 software in Microsoft Excel ${ }^{\circledR}$. The six indices applied in the first mapping were used to facilitate a taxonomic interpretation of the results. The reads that did not match with sequences of the local library were filtered in order to remove reads shorter than $140 \mathrm{bp}$ and re-mapped against the local library using the same settings and indices of the first and second mapping (M1 and M2, respectively). 
Table 1. Output parameters of de novo assembly and mapping.

\begin{tabular}{|c|c|c|c|c|c|}
\hline & & \multicolumn{2}{|c|}{ Mapping } & \multicolumn{2}{|c|}{ De novo assembling } \\
\hline & & BTL & BBM & High sensitivity & Low sensitivity \\
\hline \multirow{5}{*}{$\begin{array}{l}\text { C. albicans CMC } 1853 \\
23,810 \text { reads }\end{array}$} & $\mathrm{n}^{\circ}$ reads assembled & 23,389 & 22,755 & 23,721 & 23,674 \\
\hline & $\mathrm{n}^{\circ}$ reads not assembled & 421 & 1,055 & 89 & 136 \\
\hline & Assembly duration & 7.19 seconds & 21.58 seconds & 38 minutes- 10 seconds & 12 minutes-29 seconds \\
\hline & CPU time & 8.54 seconds & 12.49 seconds & $2 \mathrm{~h}-11$ minutes & 41 minutes- 15 seconds \\
\hline & Contigs & 1 & 1 & 119 & 219 \\
\hline \multirow{5}{*}{$\begin{array}{c}\text { C. albicans CMC } 1856 \\
53,497 \text { reads }\end{array}$} & $\mathrm{n}^{\circ}$ reads assembled & 52,270 & 51,599 & 52,187 & 53,022 \\
\hline & $\mathrm{n}^{\circ}$ reads not assembled & 1,227 & 1,898 & 1,310 & 475 \\
\hline & Assembly duration & 11.47 seconds & 33.06 seconds & 47 minutes +34 seconds & 10 minutes -46 seconds \\
\hline & CPU time & 13.37 seconds & 17.31 seconds & $2 \mathrm{~h}-49$ minutes & 34 minutes- 1 second \\
\hline & Contigs & 1 & 1 & 242 & 357 \\
\hline \multirow{5}{*}{$\begin{array}{c}\text { C. glabrata CMC } 1837 \\
19,762 \text { reads }\end{array}$} & $\mathrm{n}^{\circ}$ reads assembled & 18,883 & 18,257 & 19,454 & 19,334 \\
\hline & $\mathrm{n}^{\circ}$ reads not assembled & 879 & 1,505 & 308 & 428 \\
\hline & Assembly duration & 5.10 seconds & 21.01 seconds & 26 minutes- 10 seconds & 10 minutes- 8 seconds \\
\hline & CPU time & 6.68 seconds & 10.52 seconds & 1h-33 minutes & 34 minutes- 2 seconds \\
\hline & Contigs & 1 & 1 & 121 & 209 \\
\hline \multirow{5}{*}{$\begin{array}{c}\text { C. glabrata CMC } 1934 \\
49,337 \text { reads }\end{array}$} & $\mathrm{n}^{\circ}$ reads assembled & 47,611 & 46,352 & 48,897 & 48,479 \\
\hline & $\mathrm{n}^{\circ}$ reads not assembled & 1,726 & 2,985 & 440 & 858 \\
\hline & Assembly duration & 10.48 seconds & 34.93 seconds & $1 \mathrm{~h}-40$ minutes & 21 minutes- 11 seconds \\
\hline & CPU time & 11.48 seconds & 21.11 seconds & $5 \mathrm{~h}-20$ minutes & $1 \mathrm{~h}-11$ minutes \\
\hline & Contigs & 1 & 1 & 243 & 470 \\
\hline \multirow{5}{*}{$\begin{array}{l}\text { C. orthopsilosis CMC } 1880 \\
20,089 \text { reads }\end{array}$} & $\mathrm{n}^{\circ}$ reads assembled & 19,533 & 19,472 & 19,960 & 19,923 \\
\hline & $\mathrm{n}^{\circ}$ reads not assembled & 556 & 617 & 129 & 166 \\
\hline & Assembly duration & 7.69 seconds & 27.36 seconds & 34 minutes-27 seconds & 11 minutes- 5 seconds \\
\hline & CPU time & 10.98 seconds & 22.71 seconds & $1 \mathrm{~h}-58$ minutes & 37 minutes- 37 seconds \\
\hline & Contigs & 1 & 1 & 119 & 236 \\
\hline \multirow{5}{*}{$\begin{array}{c}\text { C. orthopsilosis CMC } 2011 \\
36,955 \text { reads }\end{array}$} & $\mathrm{n}^{\circ}$ reads assembled & 36,136 & 36,019 & 36,720 & 36,591 \\
\hline & $\mathrm{n}^{\circ}$ reads not assembled & 819 & 936 & 235 & 364 \\
\hline & Assembly duration & 11.45 seconds & 38.25 seconds & 59 minutes- 5 seconds & 15 minutes -47 seconds \\
\hline & CPU time & 7.91 seconds & 14.50 seconds & 3h-20 minutes & 51 minutes- 58 seconds \\
\hline & Contigs & 1 & 1 & 219 & 477 \\
\hline \multirow{5}{*}{$\begin{array}{c}\text { C. parapsilosis CMC } 1838 \\
37,683 \text { reads }\end{array}$} & $\mathrm{n}^{\circ}$ reads assembled & 36,717 & 36,584 & 37,683 & 37,432 \\
\hline & $\mathrm{n}^{\circ}$ reads not assembled & 966 & 1,099 & 1,264 & 251 \\
\hline & Assembly duration & 12.19 seconds & 27.66 seconds & 44 minutes- 3 seconds & 15 minutes- 11 seconds \\
\hline & CPU time & 17.55 seconds & 29.27 seconds & $2 \mathrm{~h}-33$ minutes & 51 minutes- 48 seconds \\
\hline & Contigs & 1 & 1 & 190 & 335 \\
\hline \multirow{5}{*}{$\begin{array}{c}\text { C. parapsilosis CMC } 2039 \\
23,817 \text { reads }\end{array}$} & $\mathrm{n}^{\circ}$ reads assembled & 21,549 & 21,502 & 22,569 & 22,336 \\
\hline & $\mathrm{n}^{\circ}$ reads not assembled & 2,268 & 2,315 & 1,248 & 1,481 \\
\hline & Assembly duration & 7.24 seconds & 19.88 seconds & 41 minutes- 34 seconds & 32 minutes- 57 seconds \\
\hline & CPU time & 5.73 seconds & 11.48 seconds & $2 \mathrm{~h}-24$ minutes & 1h-1 minute \\
\hline & Contigs & 1 & 1 & 281 & 632 \\
\hline \multirow{4}{*}{$\begin{array}{c}\text { C. tropicalis CMC } 2003 \\
44,744 \text { reads }\end{array}$} & $\mathrm{n}^{\circ}$ reads assembled & 41,841 & 41,361 & 43,253 & 44,140 \\
\hline & $\mathrm{n}^{\circ}$ reads not assembled & 2,903 & 3,383 & 1,491 & 604 \\
\hline & Assembly duration & 12.23 seconds & 31.09 seconds & 43 minutes- 4 seconds & 15 minutes- 9 seconds \\
\hline & CPU time & 9.70 seconds & 14.13 seconds & $2 \mathrm{~h}-28$ minutes & 45 minutes- 59 seconds \\
\hline \multirow{5}{*}{$\begin{array}{c}\text { C. tropicalis CMC } 2017 \\
20,125 \text { reads }\end{array}$} & $\mathrm{n}^{\circ}$ reads assembled & 18,007 & 17,593 & 19,671 & 19,550 \\
\hline & $\mathrm{n}^{\circ}$ reads not assembled & 2,118 & 2,532 & 454 & 575 \\
\hline & Assembly duration & 6.07 seconds & 22.98 seconds & 33 minutes- 32 seconds & 11 minutes-47 seconds \\
\hline & CPU time & 5.45 seconds & 8.12 seconds & $1 \mathrm{~h}-56$ minutes & 39 minutes- 35 seconds \\
\hline & Contigs & 1 & 1 & 369 & 527 \\
\hline
\end{tabular}


Table 1. (Continued).

\begin{tabular}{|c|c|c|c|c|c|}
\hline & & \multicolumn{2}{|c|}{ Mapping } & \multicolumn{2}{|c|}{ De novo assembling } \\
\hline & & BTL & BBM & High sensitivity & Low sensitivity \\
\hline \multirow{5}{*}{$\begin{array}{l}\text { M. guilliermondii CMC } 1825 \\
40,659 \text { reads }\end{array}$} & $\mathrm{n}^{\circ}$ reads assembled & 36,085 & 35,598 & 40,094 & 39,914 \\
\hline & $\mathrm{n}^{\circ}$ reads not assembled & 4,574 & 5,061 & 565 & 745 \\
\hline & Assembly duration & 12.25 seconds & 27.05 seconds & 51 minutes -46 seconds & 19 minutes -55 seconds \\
\hline & CPU time & 15.88 seconds & 15.43 seconds & $2 \mathrm{~h}-58$ minutes & $1 \mathrm{~h}-5$ minutes \\
\hline & Contigs & 1 & 1 & 507 & 711 \\
\hline \multirow{5}{*}{$\begin{array}{l}\text { M. guilliermondii CMC } 1924 \\
19,388 \text { reads }\end{array}$} & $\mathrm{n}^{\circ}$ reads assembled & 17,593 & 17,335 & 19,195 & 19,152 \\
\hline & $\mathrm{n}^{\circ}$ reads not assembled & 1,795 & 2,053 & 193 & 236 \\
\hline & Assembly duration & 6.33 seconds & 19.18 seconds & 22 minutes-39 seconds & 9 minutes- 18 seconds \\
\hline & CPU time & 7.02 seconds & 9.02 seconds & $1 \mathrm{~h}-20$ minutes & 31 minutes- 10 seconds \\
\hline & Contigs & 1 & 1 & 129 & 248 \\
\hline
\end{tabular}

Table 2. Type strains employed for contigs identification.

\begin{tabular}{ll} 
Species & Strain \\
\hline Candida albicans & CBS 562 \\
Candida dubliniensis & CBS 7987 \\
Candida famata & CBS 1795 \\
Candida glabrata & CBS 138 \\
Issatchenkia orientalis & CBS 573 \\
Candida metapsilosis & CBS 10907 \\
Candida orthopsilosis & CBS 10906 \\
Candida parapsilosis & CBS 604 \\
Candida pararugosa & CBS 1010 \\
Diutina rugosa & CBS 613 \\
Candida sake & CBS 159 \\
Candida tropicalis & CBS 94 \\
Candida utilis & CBS 621 \\
Candida lusitaniae & CBS 6936 \\
Meyerozyma guilliermondii & CBS 2030 \\
Saccharomyces cerevisiae* & CBS 1171 \\
*Outgroup type strain. &
\end{tabular}

\section{RESULTS}

\section{Mapping against a reference vs de novo} assembly: different efficiency in terms of time

The reads contained in a FASTQ file can be analysed with the de novo assembly or by mapping to a reference, each with a variety of algorithms and settings. The former approach does not theoretically require any a priori knowledge. Moreover, it could produce contigs of the reads without the bias due to reference sequences non including the actual species to identify. On the other hand, assembling thousands of reads deriving from hundreds of repeats without a reference could produce inaccurate assemblies. We tested these two approaches by comparing the accuracy obtained and the computation time requested. The mapping was carried out using an ad hoc library containing the ITS-LSU region of the ex-type strains of 16 yeast species, most of which are known pathogens. These two analyses were carried out on 12 strains, representative of six species and exhibiting a different number of reads, ranging from 19388 to 53 497. These analyses showed that the de novo assembly takes much more than the mapping in terms of the total time necessary to carry out the operation using an i7 Intel ${ }^{R}$ processor with $8 \mathrm{~Gb}$ RAM and the Geneious 9 interface (Table 1). The CPU time necessary for the two types of analysis diverges by two or three orders of magnitude (Fig. 1A). Within the two different approaches, BTL showed lower processing times than $\mathrm{BBm}$; the former carried out the operation with an average of 0.329 milliseconds per read, vs 0.544 milliseconds for the latter. More interestingly, the standard deviations of the BTL and BBm analyses were 0.120 and 0.226 milliseconds respectively (corresponding to 0.31 and 0.42 variation coefficients).

Using the de novo approach with the Geneious algorithm, the CPU time required was 282.38 and 91.27 milliseconds per read, with the high and low sensitivity settings (HS and LS), respectively. Even in this case, a large difference was observed between settings; the HS had a standard deviation of 91.9 milliseconds (variation coefficient 0.33 ) and the LS 34.9 standard deviation, corresponding to a 0.38 variation coefficient.

Altogether, these data indicate that the de novo assembly takes much more computational time than mapping against a reference sequence. The variability observed between the performances of four algorithms recognized in our study posed the question of the influence of the number of reads on the operational time required. Surprisingly there was a low correlation between the CPU time and the number of reads: $0.67 \mathrm{BTL}, 0.56 \mathrm{BBm}, 0.52 \mathrm{HS}$, and $0.19 \mathrm{LS}$. Finally, the correlation analysis of the computational time required by the four algorithms showed relatively high correlation values between BTL and BBm (0.741) and between the two de novo procedures (0.827) (Fig. 1B). These data indicate that the time required by mapping and assembly are independent, whereas a weak relation exists between the algorithms employed within the same type of approach.

\section{Contigs quality obtained with "mapping against a reference" and "de novo assembly"} The contigs obtained with the two methods were analysed with a local blasting using an ad hoc library containing sequences of the ex-type strains of 16 yeast species. Typical results (Fig. 2A-B) showed that high levels of 


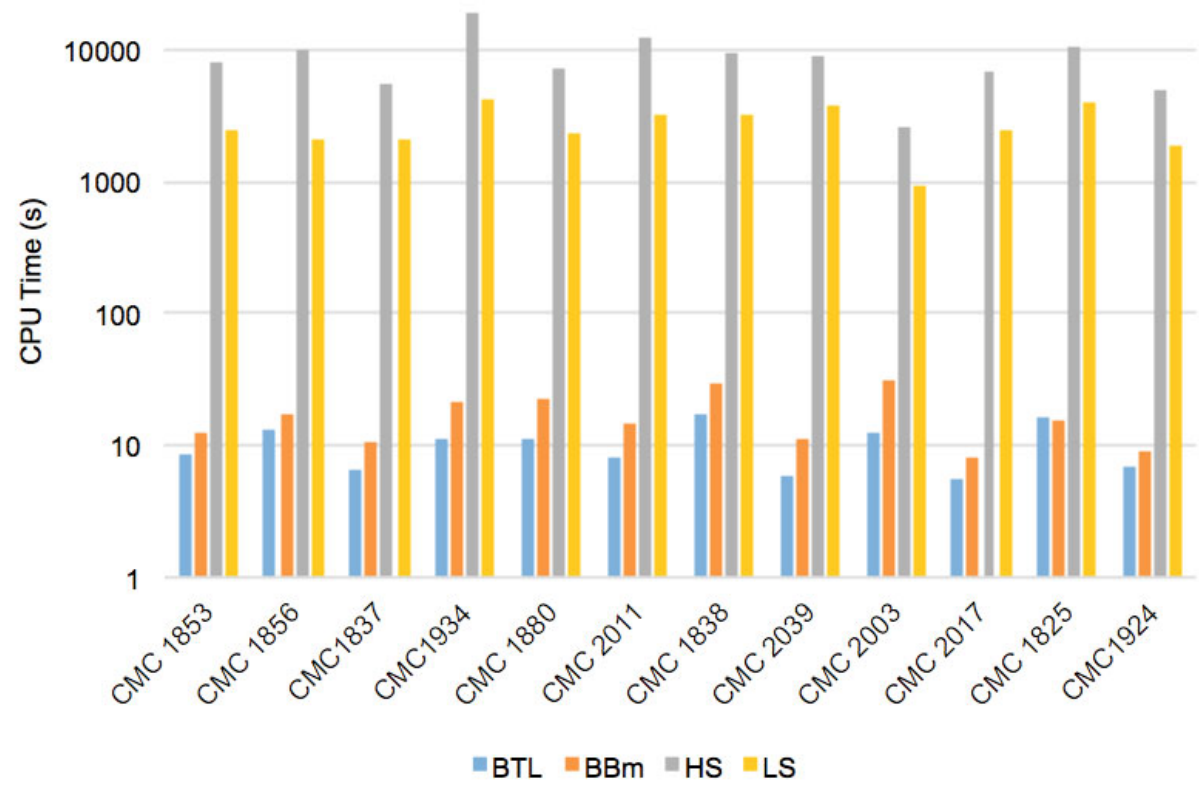

\begin{tabular}{|c|r|r|r|r|}
\hline & BTL & BBm & HS & LS \\
\hline BTL & & & & \\
\hline BBm & $\mathbf{0 . 7 4 1}$ & & & \\
\hline HS & 0.242 & 0.038 & & \\
\hline LS & 0.174 & -0.124 & $\mathbf{0 . 8 2 7}$ & \\
\hline
\end{tabular}

Fig. 1. Evaluation of the computational time requested for the two different approaches (Mapping against a Reference and De novo Assembly). A. CPU time needed by the two different types of procedures with the four settings. B. Correlation between the four different algorithms.

nucleotide similarity were obtained between the single contig of mapping with most of the library members. These nucleotide similarities spanned from approximately $80 \%$ to $99.6 \%$ of the correctly identified species (Fig. 2A). In this analysis no major differences were observed between the BTL and the BBm algorithms. On the contrary, blasting the several contigs derived from the de novo assembly produced different putative identifications with homologies spanning from approximately almost $0 \%$ to $13 \%$, whereas the correct species displayed $69.4 \%$ and $74.6 \%$ homology with the high and low sensitivity algorithms. These types of results deriving from the two approaches were confirmed for all the strains analysed. For the mapping approach, the similarities with the correct species were in the range between $97.88 \%$ and $99.8 \%$, whereas the homology shown with the second more similar species varied between $89.9 \%$ and $98.8 \%$ using BTL and BBm algorithms (Fig. 2C). The blasting of the contigs of the 12 strains obtained with de novo assembly gave homologies with the correct species ranging from $52.4-87.5 \%$ and from $48.6-90.5 \%$ with the high and the low sensitivity algorithms, respectively (Fig. 2D). The second most similar species showed homologies in the ranges $3.8-38 \%$ and $3.8-34 \%$, respectively with the HS and LS algorithms.

\section{Feasibility of "mapping against a reference" with large libraries}

From the above results, it was clear that the use of de novo assembly is time consuming and produced relatively low homologies to the expected species. These two aspects suggested a more detailed analysis of the data to determine the possibilities offered by the mapping when the reference is represented by a large library of sequences. Ideally, these reference sequences should contain the sequences from the type strains of all known species, in order to avoid the presence of misidentified strains that would lead not only to an incorrect identification, but to an inflation of misidentifications. For this reason, ad hoc, highly curated libraries are produced and maintained, such as the Westerdijk Fungal Biodiversity Institute (http://www.westerdijkinstitute.nl/), a fungal reference library (RefSeq) within the NIH-GenBank (Schoch et al. 2014), or the dedicated ITS library of ISHAM for human and animal pathogenic fungal identifications (Irinyi et al. 2015).

In order to test the efficacy of mapping against a reference library using large collections of sequences, we used three libraries of different size: (1) a curated library of type strains from the Westerdijk Fungal Biodiversity Institute (CBS) containing only ITS sequences (15 565 sequences); (2) a curated library with both ITS and LSU D1/D2 sequences from Westerdijk Fungal Biodiversity Institute (CBS) (34 683 sequences); and (3) the ISHAM-ITS database (2727 sequences). The strains CMC 1793 and CMC 1818 with 21 


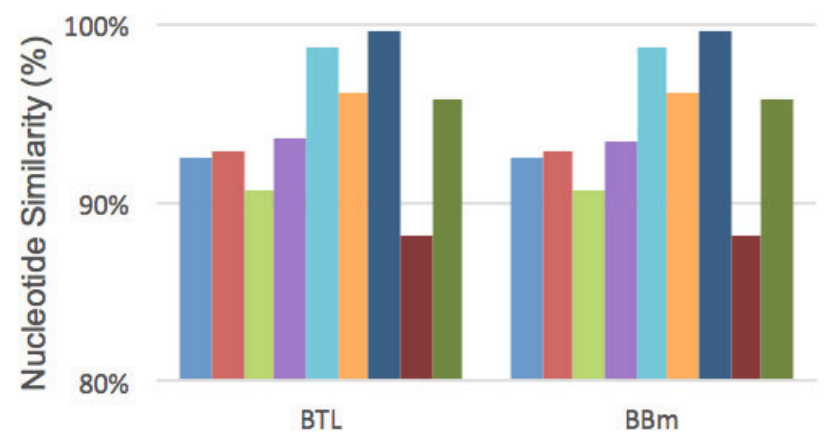

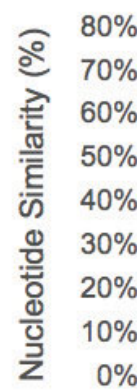

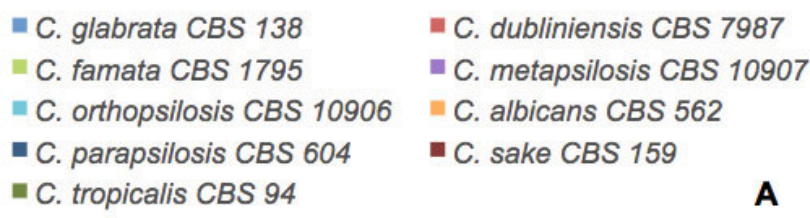

A



\section{- C. albicans CBS 562 \\ I. orientalis CBS 573 \\ - C. glabrata CBS 138 \\ C. metapsilosis CBS 10907 \\ C. orthopsilosis CBS 10906 C. parapsilosis CBS 604 \\ C. rugosa CBS 613 \\ C. tropicalis CBS 94}

C

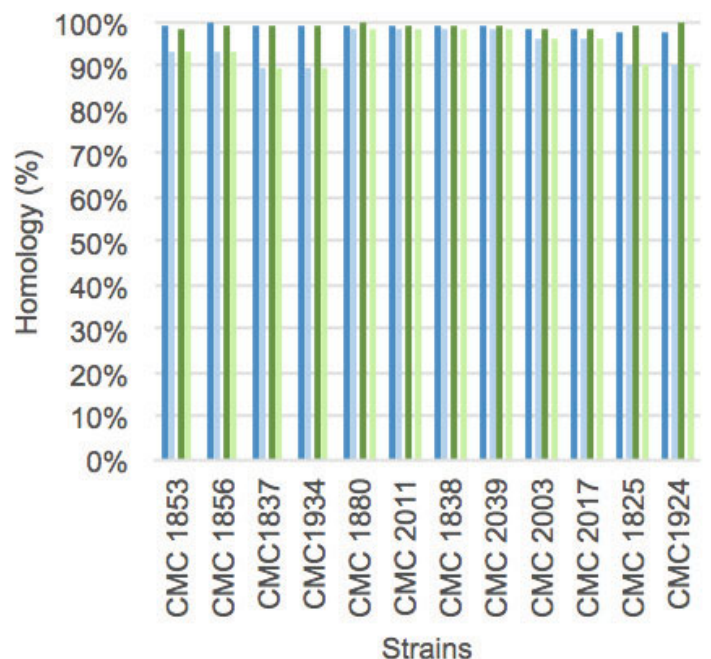

Fig. 2. Analysis of contigs quality. A. Similarity between a single contig of each of the two mapping algorithms and the members of the reference library. B. Variation of the similarity with the members of the library using High Sensitivity (HS) or Low Sensitivity (LS) algorithms. C. Homology of the contigs with the first and the second most similar species using BTL (dark-light blue) and BBm (dark-light green). D. Homology of the contigs analysed with High Sensitivity (dark-light blue) or Low Sensitivity (dark-light green) algorithms.

238 and 58263 reads respectively were mapped against the three libraries using BTL and BBm algorithms. This scheme produced 12 mapping combinations that were tested in order to define the feasibility of using large libraries and these performance parameters.

Using an i7 Intel processor with $8 \mathrm{~Gb}$ Ram and the Geneious 9 interface, the minimum CPU time was $13.59 \mathrm{~s}$ and the maximum $393 \mathrm{~s}$. The average time for CMC 1793 mapping was $52.65 \mathrm{~s}$, whereas CMC 1818 (with almost three times more reads) required an average time of $131.02 \mathrm{~s}$. The time performances of the three libraries varied, as expected, according to their size, expressed as number of sequences. The relatively small ISHAM-ITS library required an average of $22.31 \mathrm{~s}$, whereas the average CPU times of the two Westerdijk Fungal Biodiversity Institute (CBS) libraries were 61.43 and $191.75 \mathrm{~s}$ for the Westerdijk Fungal Biodiversity
Institute CBS-ITS and Westerdijk Fungal Biodiversity Institute CBS ITS-LSU.

The time performance of the two tested algorithms changed according to the size of both the library and the FASTQ file (Fig. 3A). BBm was faster than BTL, especially when processing the large CMC 1818 file with over 58000 reads, whereas it was slightly slower with the smaller FASTQ file from CMC 1793. The processing rate was obviously conditioned by the library size as well. Both algorithms showed the largest reads/second values with the ISHAMITS and the smallest with the very large Westerdijk Fungal Biodiversity Institute CBC ITS-LSU library. Taking together these observations, we tested the hypothesis that the reads/ sec. processing rate may be function of the number of reads of the FASTQ file and the number of sequences of the library used. The regression analysis, carried out for BTL and BBm 

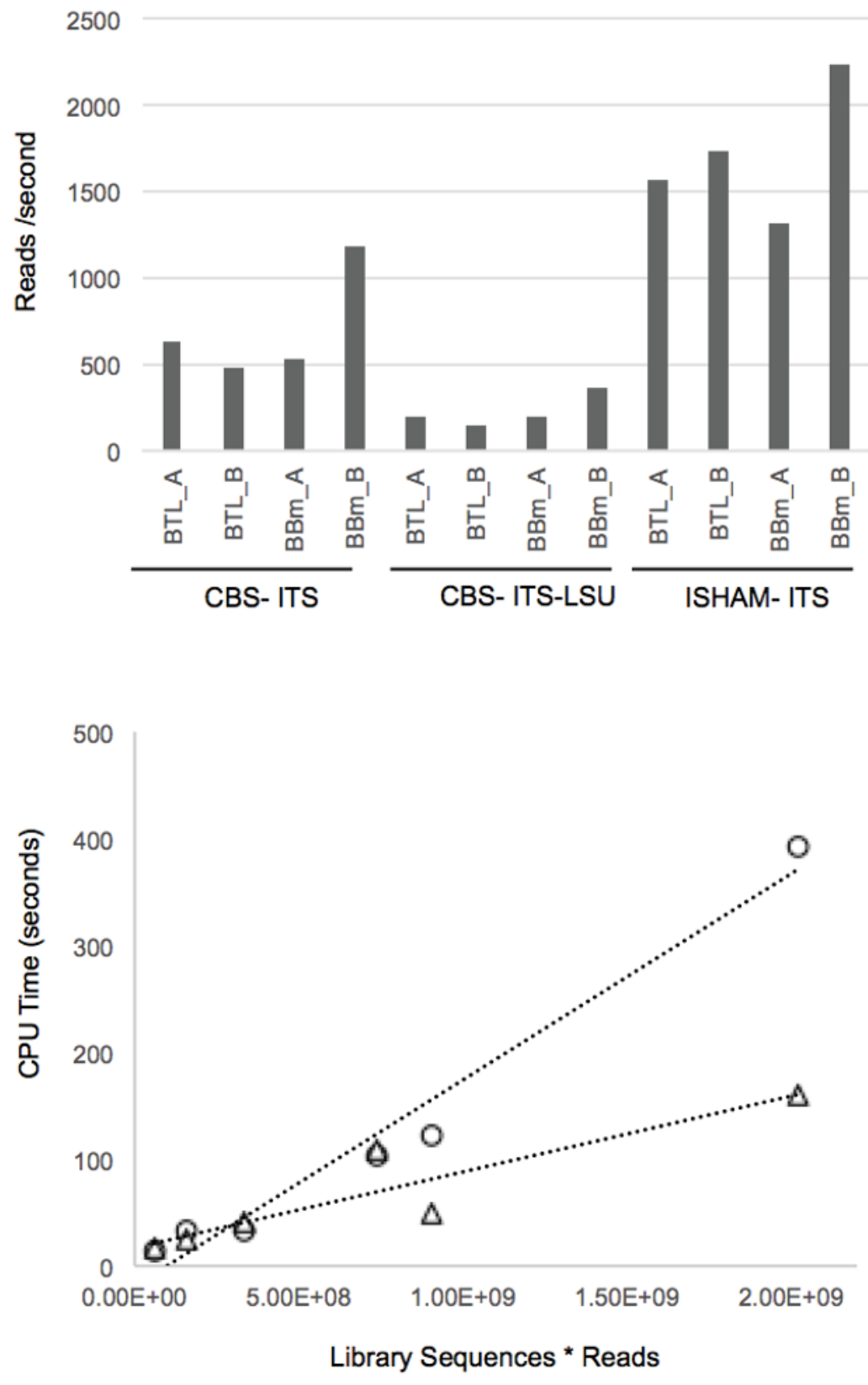

Fig. 3. Time performance of the mapping algorithms against three large libraries. A. Variation of the time performance using references and files of different size. B. Regression analysis between the time performance, the dimension of library and FASTQ files using the BTL (circle) and $\mathrm{BBm}$ (triangle) algorithms. separately, yielded 0.9702 and $0.8354 \mathrm{R} 2$ values for BTL and BBm, respectively (Fig. $3 \mathrm{~B}$ ). Taken together, these data indicated that mapping against a reference is feasible in terms of time and that it is more convenient than de novo assembly, even if large libraries and FASTQ files are employed. This analysis showed that the use of a large library as reference returns homology values ranging from $60-100 \%$ (Table 3), indicating that a careful analytical protocol is necessary to discriminate the taxonomically positive identifications.

\section{A pipeline to optimize the mapping against a reference}

The tests described above showed that, even using large libraries, mapping is faster than de novo assembling. Furthermore, the levels of homology typically found with Sanger sequencing are closer to the data yielded by mapping than those produced by de novo assembling. These characteristics suggested the development of a pipeline to take into consideration all aspects emerging from our tests and accounting for the typical conditions in which identifications are carried out. The first step is mapping against a reference (mapping M1) to indicate the most likely species to which the strain belongs. Since mappings can be used with a wide range of parameters and their examination is quite difficult, especially when the libraries and the FASTQ files are large, we developed a series of indices, including a final synthetic index Isyn (Table $4 \mathrm{~A}-\mathrm{B}$ ). These indices are reported as percentages, for easier reading. All the calculated indices are consistently higher with the expected species (e.g C. glabrata in Table 4A) as indicated by standard deviations not higher than $1.5 \%$. The reads of the conserved sequences are often very similar to more than one member of the library. This produces a biased decrease of all indices of the species to whom the unknown strain is expected to be attributed. This means that when the library increases in 
Table 3. Performances of algorithms in mapping FASTQ files of different size with large reference libraries.

\begin{tabular}{|c|c|c|c|c|c|c|c|c|c|c|c|}
\hline \multicolumn{2}{|c|}{ Library } & \multirow[t]{2}{*}{ Algorithm } & \multicolumn{2}{|c|}{ FASTQ } & \multicolumn{2}{|c|}{ Time Performances } & \multicolumn{5}{|c|}{ Mapping parameters } \\
\hline acronym & sequences & & strain & reads & $\begin{array}{l}\text { Mapping } \\
\text { time (sec) }\end{array}$ & $\begin{array}{l}\text { CPU-time } \\
\text { (sec) }\end{array}$ & used reads & unused reads & Matches & $\begin{array}{l}\min \\
\text { pairwise } \\
\text { identities }\end{array}$ & $\begin{array}{l}\text { max } \\
\text { pairwise } \\
\text { identities }\end{array}$ \\
\hline & 15,565 & BTL & A & 21,238 & 63 & 33,34 & 18,274 & 2,964 & 684 & $74,00 \%$ & $100 \%$ \\
\hline CBS & & & B & 58,263 & 99 & 123 & 54,822 & 3,441 & 661 & $91,00 \%$ & $100 \%$ \\
\hline \multirow[t]{3}{*}{ ITS } & & $\mathrm{BBm}$ & A & 21,238 & 80 & 39,82 & 18,323 & 2,915 & 626 & $68,20 \%$ & $100 \%$ \\
\hline & & & B & 58,263 & 114 & 49,57 & 55,675 & 2,588 & 708 & $62,30 \%$ & $100 \%$ \\
\hline & 34,683 & BTL & A & 21,238 & 186 & 104 & 19,163 & 2,075 & 2,051 & $73,40 \%$ & $100 \%$ \\
\hline CBS & & & B & 58,263 & 273 & 393 & 57,140 & 1,123 & 2,457 & $79,50 \%$ & $100 \%$ \\
\hline \multirow[t]{3}{*}{ ITS-LSU } & & $\mathrm{BBm}$ & A & 21,238 & 204 & 109 & 19,000 & 2,238 & 1,939 & $58,80 \%$ & $100 \%$ \\
\hline & & & B & 58,263 & 311 & 161 & 56,930 & 1,333 & 2,445 & $63,60 \%$ & $100 \%$ \\
\hline & 2,727 & BTL & A & 21,238 & 16,7 & 13,59 & 13,904 & 7,334 & 319 & $74,10 \%$ & $100 \%$ \\
\hline ISHAM & & & B & 58,263 & 30,3 & 33,5 & 36,416 & 21,847 & 297 & $82,00 \%$ & $100 \%$ \\
\hline \multirow[t]{2}{*}{ ITS } & & $\mathrm{BBm}$ & A & 21,238 & 37,7 & 16,13 & 13,665 & 7,573 & 280 & $74,50 \%$ & $100 \%$ \\
\hline & & & B & 58,263 & 45,2 & 26,03 & 35,653 & 22,610 & 305 & $85,70 \%$ & $100 \%$ \\
\hline
\end{tabular}

Strain A: CMC 1793; strain B: CMC 1818.

size, and includes a large number of entries similar to the strain under identification, all indices and the Isyn will show relatively low values. The M1 mapping does not yield a definitive identification at the species level, but rather gives an indication of the most likely species and the ex-type strain that should be used in the next M2 mapping as reference. M2 produces the same parameters as $\mathrm{M} 1$ and normally sets aside a relatively number of "unused reads" that usually range from $5 \%$ to over about $30 \%$. Some of these reads were highly homologous (i.e. $>98 \%$ homology) to the rDNA of other species. These considerations led us to propose a third mapping (M3) similar to $\mathrm{M} 1$ in which all the unused reads are mapped against the same selected library. To ease the reading of the output parameters the indices were calculated on the outputs of M2 and M3 jointly (Table 4B). The major difference between the $\mathrm{M} 1$ and the M2-M3 mappings is that the conserved sequences are attributed to the most likely species in the latter, whereas they are distributed randomly and evenly in the former. The whole M2-M3 procedure led to homology values comparable to those usually observed with Sanger sequencing.

These findings suggest a pipeline, in which the preliminary attribution to species is carried out with M1. An alternative to the use of $M 1$ is the case in which the microbiologist has a some evidence to restrict the identification to one or few presumptive specis. However, if even after M2-M3 mapping a high level of homology with the ex-type strain of a known species is not reached, the identification should be questioned and the possibility that the strain represents a new species should be considered. Residual unused reads after M2-M3 mapping, can be considered either as background noise or requiring further investigation.

\section{Validation of the procedure with a large group of strains}

Our procedure was tested with 286 strains, isolated as opportunistic pathogenic yeasts from patients at two Italian hospitals. Both BTL and BBm algorithms were used. Since the yeasts were supposedly part of the known pathogenic yeasts attributed to Candida, a restricted reference library of the 16 ex-type strains of species accepted in this genus was employed. The analyses produced output values with the members of the library that led to the calculation of the indices, including Isyn (Table 4). For simplicity, the identification characterized by the highest Isyn will be hereinafter referred to as a "correct identification", and the others as "incorrect identifications".

It must be noted that the Isyn value gives an overview of the homology of the reads in a FASTQ file with the members of the library. Even a high Isyn value does not preclude that some of the reads show a high homology with an ex-type strain not representing the species, which the unknown strain belongs to. This is, for instance, the case of the C. glabrata CMC 1912 strain, which included some $2.86 \%$ of the reads with over $98 \%$ of homology with C. albicans.

Results of the BTL mapping showed that the majority of the "correct identifications" ranged from 80-95\% and no strain showed an Isyn higher that $95 \%$ in the M1 mapping. The majority of the M2-M3 mapping results were in the range 95-100 \%, with only $10 \%$ of the strains showing less than $95 \%$ Isyn (Fig. 4A). Similar results were obtained with the BBm algorithm, although some $4 \%$ of the strains had an Isyn higher than $95 \%$ and only around $5 \%$ of the strains had less than $95 \%$ Isyn with M2-M3 mapping (Fig. 4B).

The distribution of the "incorrect identifications" was studied with both algorithms, showing a decrease of their maximum frequency from $I s y n=20 \%$ to $I s y n=5 \%$ respectively, with the M1 and M2-M3 mappings (Fig. 4C-D).

One of the major differences between the $\mathrm{M} 1$ and the M2-M3 mapping results is that in the former most of the reads display a very high homology (> $95 \%$ ) with the sequences contained in the library, whereas in the latter this frequency decreases to around $5 \%$ (Table 4A-B). This was observed in all the analysed strains, and indicates that M1 alone can produce highly biased results, especially if 
Table 4. Example of a M1 and M2-M3 mapping against an ad hoc library of 16 ITS-LSU concatenate rDNA sequences of type strains of pathogenic yeasts (CMC 1912 strain).

A

\begin{tabular}{|c|c|c|c|c|c|c|c|c|c|c|c|}
\hline \multirow[t]{2}{*}{ Mapping M1 Algorithm: BTL } & & \multirow{2}{*}{$\begin{array}{l}\text { Mapping } \\
\text { \# Sequences }\end{array}$} & \multicolumn{9}{|l|}{ parameters } \\
\hline & \# Nucleotides & & $\%$ Of Ref Seq & $\begin{array}{l}\% \text { Pairwise } \\
\text { Identity }\end{array}$ & Mean Cover. & Iread & Inuc & Icov & Iref & Isim & Isyn \\
\hline C. albicans & 212,814 & 1,426 & $99,90 \%$ & $99,00 \%$ & 203,48 & $6,14 \%$ & $6,14 \%$ & $9,26 \%$ & $6,14 \%$ & $6,08 \%$ & $6,80 \%$ \\
\hline C. dubliniensis & 12,362 & 80 & $53,20 \%$ & $99,30 \%$ & 10,01 & $0,34 \%$ & $0,36 \%$ & $0,46 \%$ & $0,19 \%$ & $0,35 \%$ & $0,34 \%$ \\
\hline C. famata & 1,863 & 6 & $24,40 \%$ & $98,80 \%$ & 0,56 & $0,03 \%$ & $0,05 \%$ & $0,03 \%$ & $0,01 \%$ & $0,05 \%$ & $0,03 \%$ \\
\hline C. glabrata & $3,004,133$ & 20,223 & $100,00 \%$ & $99,00 \%$ & 1813,15 & $87,06 \%$ & $86,74 \%$ & $82,51 \%$ & $86,74 \%$ & $85,87 \%$ & $86,41 \%$ \\
\hline I. orientalis & 2,689 & 12 & $42,20 \%$ & $96,60 \%$ & 1,3 & $0,05 \%$ & $0,08 \%$ & $0,06 \%$ & $0,03 \%$ & $0,07 \%$ & $0,06 \%$ \\
\hline C. metapsilosis & 3,886 & 20 & $43,10 \%$ & $99,00 \%$ & 1,93 & $0,09 \%$ & $0,11 \%$ & $0,09 \%$ & $0,05 \%$ & $0,11 \%$ & $0,09 \%$ \\
\hline C. orthopsilosis & 8,590 & 52 & $92,30 \%$ & $99,10 \%$ & 6,34 & $0,22 \%$ & $0,25 \%$ & $0,29 \%$ & $0,23 \%$ & $0,25 \%$ & $0,25 \%$ \\
\hline C. parapsilosis & 70,672 & 468 & $99,10 \%$ & $96,90 \%$ & 61,27 & $2,01 \%$ & $2,04 \%$ & $2,79 \%$ & $2,02 \%$ & $1,98 \%$ & $2,18 \%$ \\
\hline C. pararugosa & 0 & 0 & 0 & 0 & 0 & $0,00 \%$ & $0,00 \%$ & $0,00 \%$ & $0,00 \%$ & $0,00 \%$ & $0,00 \%$ \\
\hline D. rugosa & 1,034 & 2 & $4,50 \%$ & $92,90 \%$ & 0,05 & $0,01 \%$ & $0,03 \%$ & $0,00 \%$ & $0,00 \%$ & $0,03 \%$ & $0,01 \%$ \\
\hline C. sake & 105,986 & 703 & $35,00 \%$ & $99,00 \%$ & 69,76 & $3,03 \%$ & $3,06 \%$ & $3,17 \%$ & $1,07 \%$ & $3,03 \%$ & $2,69 \%$ \\
\hline C. tropicalis & 23,711 & 154 & $96,40 \%$ & $99,20 \%$ & 20,51 & $0,66 \%$ & $0,68 \%$ & $0,93 \%$ & $0,66 \%$ & $0,68 \%$ & $0,73 \%$ \\
\hline C. utilis & 1,705 & 5 & $14,70 \%$ & $99,70 \%$ & 0,53 & $0,02 \%$ & $0,05 \%$ & $0,02 \%$ & $0,01 \%$ & $0,05 \%$ & $0,03 \%$ \\
\hline C. Iusitaniae & 970 & 2 & $12,70 \%$ & $100,00 \%$ & 0,13 & $0,01 \%$ & $0,03 \%$ & $0,01 \%$ & $0,00 \%$ & $0,03 \%$ & $0,01 \%$ \\
\hline M. guilliermondii & 8,425 & 52 & $97,70 \%$ & $98,90 \%$ & 6,21 & $0,22 \%$ & $0,24 \%$ & $0,28 \%$ & $0,24 \%$ & $0,24 \%$ & $0,25 \%$ \\
\hline S. cerevisiae & 4,642 & 25 & $19,00 \%$ & $99,20 \%$ & 2,32 & $0,11 \%$ & $0,13 \%$ & $0,11 \%$ & $0,03 \%$ & $0,13 \%$ & $0,10 \%$ \\
\hline
\end{tabular}

B

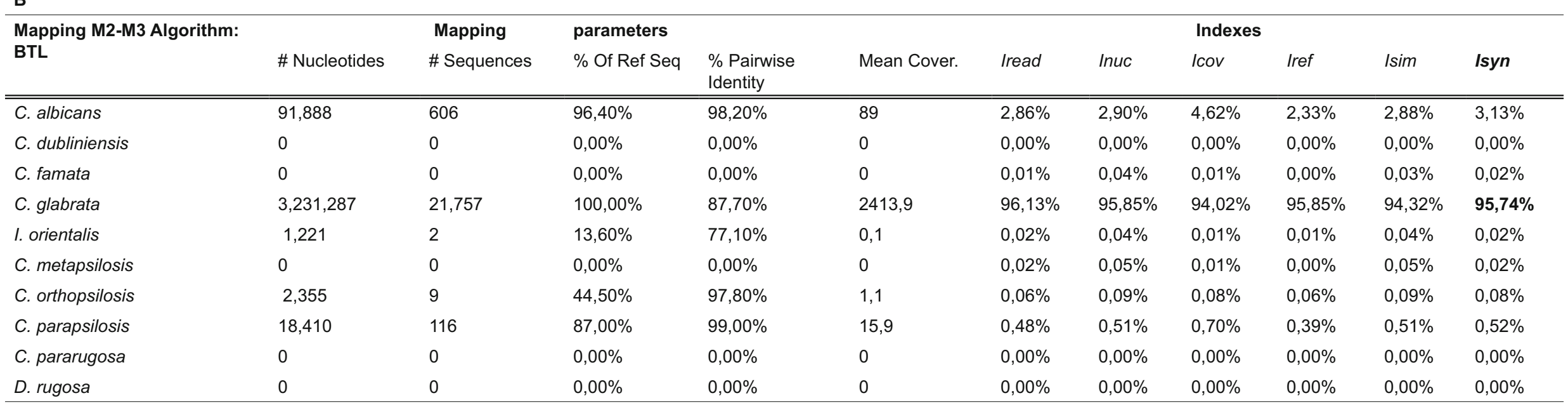




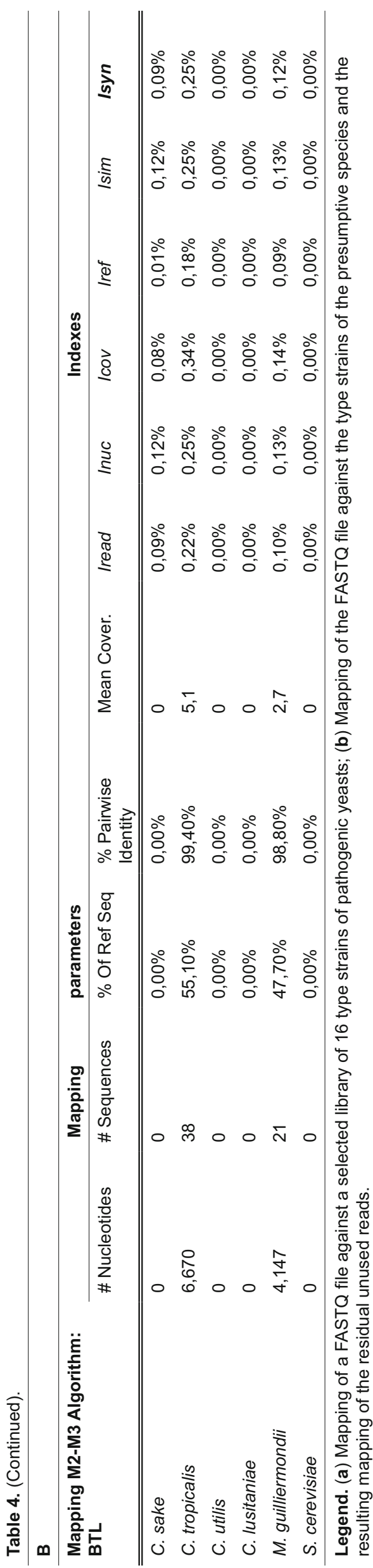

the homology (e.g. pairwise identity) is used directly without any other correction. Altogether, it seems that the Isyn from the M2M3 mapping produces data reliable and quite comparable to the Sanger homology levels commonly used by taxonomists for strain identification.

The reads with high homology (> $97 \%$ ) to one of the library's ex-type strains were $23.96 \%$ with BTL Local and $18.31 \%$ with $\mathrm{BBm}$. These relatively high frequencies could be due to the similarity among the members of the library, because similar references would share most of the common conserved regions. In order to test this hypothesis, we analysed the behaviour of the strains belonging to the sister species C. parapsilosis and C. orthopsilosis. These strains had $23.97 \%$ and $20.64 \%$ reads with $<97 \%$ of homology, using BTL and BBm, respectively; this indicates that the "incorrect identifications" would be increased by libraries containing highly related ex-type strains, when using the M1 mapping.

\section{Different estimates of the internal variability among rDNA copies}

Our results show that M1 mapping of NGS reads detects the variability intrinsic among the rDNA copies, generating possible misclassifications. Analysis of the data from all M1 mappings showed that $28.6 \%$ and $24.1 \%$ average internal heterogeneity was detected by BTL and BBm (Fig. 5). This heterogeneity ranged from $14.7-45.7 \%$ with $B T L$, and from $24.1-42.8 \%$ with $\mathrm{BBm}$. These data indicate that the M1 mapping can only indicate which is the most probable species of the unknown isolate, but cannot determine its precise similarity to the ex-type strain. The subsequent M2 mapping allows the determination of this similarity at a good level, and produces a marked reduction in heterogeneity, averaging $3.4 \%$ and $2.9 \%$ for $\mathrm{BTL}$ and $\mathrm{BBm}$, respectively. However, the application of M2 still detects up to $6.1 \%$ (BTL) and $7 \%$ heterogeneity $(\mathrm{BBm})$.

These analyses showed that the sole application of $M 1$ mapping produced a high rate of "incorrect identifications", generating over $20 \%$ of false positive identifications, that can be effectively corrected by M2-M3 mapping.

\section{DISCUSSION}

The increasing expansion of NGS and the unparalleled wealth of output reads occurring with rapidly decreasing prices, necessitated a consideration of this technology in the identification of fungal strains. There are currently two different scenarios in which identification to species level is required, the classical identification of an isolate, and the massive identification of the members of a population by direct sequencing. The latter approach, metagenomics, is technically bound to NGS, whereas the identification of an isolate can be carried out with both Sanger sequencing and NGS. The cost and the throughput possibilities of NGS are already more competitive than the traditional Sanger sequencing in many analytical settings, bringing the possibility of employing NGS in both scenarios. At this time, the molecular identification of yeasts and filamentous fungi is carried out with markers included in the chromosomal region encoding the ribosomal RNA (Schoch et al. 2012). The advantage of these markers is the ease of manipulation due to the amount of DNA present 



Fig. 4. Distribution of the similarity to ex-type strains with different analytical combinations. A. BTL Local - similarity to the correct species. B. $\mathrm{BBm}$ - similarity to the correct species. C. BTL Local - similarity to the incorrect species. D. BBm - similarity to the incorrect species. Light Red = mapping M1; Light Blue = mappings M2 and M3; Yellow = mapping M1; Green = Mappings M2 and M3.



\section{Algorithms applied}

Fig. 5. Estimation of the internal variability among the rDNA copies. 
in the cell, since this region represents a large fraction of the genome (Dujon et al. 2004). Not less important is that the tandem structure, with the alternation of conserved and variable regions, facilitates location of the anchoring places for primers and that both the LSU and the ITS regions are within the reach of a normal Sanger run. On the other hand, these markers belong to a large multigene family in which the presence of so many copies that are not necessarily identical poses some problems when using NGS both for strain identification and metagenomics. The dual aims of this paper were to find a rapid and reliable pipeline to employ NGS in strain identification, and to estimate the effects of internal heterogeneity among copies on the quality of the identification in a metagenomic scenario.

For single strain identification, the proposed pipeline was shown to have the rapidity to massively identify or re-identify thousands of strains in matter of days, with a very high level of accuracy arising from the high number of reads. This makes the final sequence several hundred times deeper than the Sanger ones that have a maximum depth of two in most routine applications. Of course, the high depth combined with the heterogeneity required the three mappings described here, which are not in contrast with the rapidity of the data processing. Considering the number of reads deriving from a single NGS analysis, and the relatively short length of the amplicons, this approach has the potential for multiplexing. The technique allowed the sequencing of two different marker loci, the LSU gene and ITS region, that represent a low level of multiplexing, although the depth obtained indicated that there is room for more markers (e.g. TEF1-alpha, RPB1, RPB2) (Kurtzman \& Robnett 2013), especially if not represented by multi-copy genes, as was the case here. The use of Multi Locus Sequencing has been recommended for the superior ability to detect the species circumspection and is particularly important when the taxa are phylogenetically close and therefore require a high level of taxonomic resolution. Still, the markers proposed (Kurtzman \& Robnett 2013) present some practical problems, such as the difficulty of finding universal anchoring positions for both the primary amplification and the sequencing, making their use not as straightforward as one would expect in routine procedures for identification or, even more, diagnostics.

Our initial hypothesis was that, in the case of multigene families, the sequencing depth obtainable with ampliconbased NGS could produce information on the real extent of the internal heterogeneity, possibly masked by the peculiarities of Sanger sequencing. This aspect is particularly relevant to predict the behaviour of heterogeneous multigene families, as the rRNA encoding genes, when subject to NGS for the direct exploration of environmental biodiversity, taking a metagenomic culture-independent approach, to explore the effects of the rDNA heterogeneity in metagenomic identifications. For this reason, we carried out identifications at the strain level with a technique amenable of working in metagenomics with DNA derived from an unknown number of taxa. The results indicated that the relatively high heterogeneity within a single genome must be taken into account in case NGS is used to sequence multigene markers for identification, both at the strain and metagenomics level.
In the first case, the simple usage of the procedure described allows an accurate determination of the similarity of the strain with the ex-type of the species it belongs to. Moreover, the application of M2-M3 mappings yields similarity values comparable with those produced by Sanger sequencing, allowing to use the same threshold values proposed in studies carried out using Sanger sequencing data ( Vu et al. 2016).

In general, our work point out that some sort of pipeline is necessary to ensure a "correct" species identification and that mapping is much more efficient than assembly. These observations are in contrast with those proposed by Ahmed (2016) using data from a different platform and experimental design, and indicate that some sort of pipeline is necessary to decrease the extent of misidentification in metagenomics settings.

The heterogeneity with M1 mapping was an average of $21.13 \%$ throughout all the species considered. When M2M3 mappings were sequentially applied, the number of reads not homologous to the species of appurtenance dropped to around $2.1 \%$. The latter estimate of internal heterogeneity is quite close to the ca. $3 \%$ expected (Lindner et al. 2013). These figures mean that the actual heterogeneity is indeed around $3 \%$, which seems to support more the classical theory of gene conversion (Ganley \& Kobayashi 2007). The problem in metagenomics is that the variability would be estimated at around $20 \%$ because the M2-M3 mappings can only be applied to the scenario of isolated strains, and not in metagenomics. This is due to the number of species present in a metagenomics sample being unknown, whereas a single isolate can belong only to one species, allowing the redistribution of the various reads not attributed directly to the right taxon. A way around this problem would be to extend this type of study to other taxa in order to: (1) verify the extent of the internal variability of the rDNA region; and (2) define an algorithm that disregards all taxa below a given threshold to eliminate artefacts, i.e. reads that at the single strain level would not be attributed to the "correct" species.

Further works, similar to that presented here, will be needed to determine whether these thresholds are similar in different groups of fungi, and would in also allow for a correction of the alpha-diversity estimate that could be produced by an unsupervised usage of NGS, i.e. by automatic pipelines without the actual supervision of a microbiologist.

Taking these considerations together, we conclude that the possibilities offered by current NGS techniques, and their future developments, promise to shed more light on rDNA composition and to transform its internal variability into a powerful taxonomic tool.

\section{ACKNOWLEDGEMENTS}

CC was supported by a PhD program of the Biotechnology Doctorate at the University of Perugia paid by MIUR (Ministero dell'Istruzione, dell'Università e della Ricerca). LR was supported as temporary researcher with a grant from Fondazione Cassa di Risparmio di Perugia. Partially supported by the GILEAD (Gilead Sciences, Inc.,) Fellowship Programme: "Caratterizzazione del biofilm ed efficacia del trattamento con Amfotericina $B$ liposomiale in ceppi filmogeni del 
gruppo Candida parapsilosis sensu lato". WM and VR are supported by an Australian NH\&MRC (National Health and Medical Research Council) grant \#APP1121936. The authors acknowledge gratefully Angela Conti for friendly technical help.

\section{REFERENCES}

Ahmed A (2016) Analysis of metagenomics Next Generation Sequence data for fungal ITS barcoding: do you need advance bioinformatics experience? Frontiers in Microbiology 7: 1061.

Alper I, Frenette M, Labrie S (2011) Ribosomal DNA polymorphisms in the yeast Geotrichum candidum. Fungal Biology 115: 12591269.

Amend AS, Seifert KA, Bruns TD (2010) Quantifying microbial communities with 454 pyrosequencing: does read abundance count? Molecular Ecology 19: 5555-5565.

Berrocal CA, Rivera-Vicens RE, Nadathur GS (2016) Draft genome sequence of the heavy-metal-tolerant marine yeast Debaryomyces hansenii J6. Genome announcements 4: e00983-00916.

Bokulich NA, Mills DA (2012) Next-generation approaches to the microbial ecology of food fermentations. BMB Reports 45: $377-$ 389.

Bushnell B (2014) BBMap: a fast, accurate, splice-aware aligner. Berkeley: Ernest Orlando Lawrence Berkeley National Laboratory.

Corte L, Roscini L, Colabella C, et al. (2016) Exploring ecological modelling to investigate factors governing the colonization success in nosocomial environment of Candida albicans and other pathogenic yeasts. Scientific Reports 6: 26860.

Dujon B (1996) The yeast genome project: what did we learn? Trends in Genetics 12: 263-270.

Dujon B, Sherman D, Fischer G, et al. (2004) Genome evolution in yeasts. Nature 430: 35-44.

Ganley AR, Kobayashi T (2007) Highly efficient concerted evolution in the ribosomal DNA repeats: total rDNA repeat variation revealed by whole-genome shotgun sequence data. Genome Research 17: 184-191.

Gong J, Dong J, Liu X (2013) Extremely high copy numbers and polymorphisms of the rDNA operon estimated from single cell analysis of oligotrich and peritrich ciliates. Protist 164: 369-379.

Groenewald M, Robert V, Smith MT (2011) The value of the D1/D2 and internal transcribed spacers (ITS) domains for the identification of yeast species belonging to the genus Yamadazyma. Persoonia 26: $40-46$.

Hajibabaei M (2012) The golden age of DNA metasystematics. Trends in Genetics 28: 535-537.

Imabayashi Y, Moriyama M, Takeshita T, et al. (2016) Molecular analysis of fungal populations in patients with oral candidiasis using next-generation sequencing. Scientific Reports 6: 28110.

Irinyi L, Serena C, Garcia-Hermoso D, et al. (2015) International Society of Human and Animal Mycology (ISHAM)-ITS reference DNA barcoding database-the quality controlled standard tool for routine identification of human and animal pathogenic fungi. Medical Mycology: myv008.

Jones T, Federspiel NA, Chibana H, et al. (2004) The diploid genome sequence of Candida albicans. Proceedings of the National Academy of Sciences, USA 101: 7329-7334.

Kearse M, Moir R, Wilson A, et al. (2012) Geneious Basic: an integrated and extendable desktop software platform for the organization and analysis of sequence data. Bioinformatics 28 : 1647-1649.

Korabecna M (2007) The variability in the fungal ribosomal DNA (ITS1, ITS2, and 5.8 S rRNA gene): its biological meaning and application in medical mycology. Communicating Current Research and Educational Topics and Trends in Applied Microbiology 105: 783-787.

Kurtzman CP, Robnett CJ (1998) Identification and phylogeny of ascomycetous yeasts from analysis of nuclear large subunit (26S) ribosomal DNA partial sequences. Antonie van Leeuwenhoek 73: 331-371.

Kurtzman CP, Robnett CJ (2013) Relationships among genera of the Saccharomycotina (Ascomycota) from multigene phylogenetic analysis of type species. FEMS Yeast Research 13: 23-33.

Langmead B, Salzberg SL (2012) Fast gapped-read alignment with Bowtie 2. Nature Methods 9: 357-359.

Li W, Sun H, Deng Y, Zhang A, Chen H (2014). The heterogeneity of the rDNA-ITS sequence and its phylogeny in Rhizoctonia cerealis, the cause of sharp eyespot in wheat. Current Genetics 60: 1-9.

Liao D (1999) Concerted evolution: molecular mechanism and biological implications. American Journal of Human Genetics 64: 24-30.

Lindner DL, Banik MT (2011) Intragenomic variation in the ITS rDNA region obscures phylogenetic relationships and inflates estimates of operational taxonomic units in genus Laetiporus. Mycologia 103: 731-740.

Lindner DL, Carlsen T, Henrik Nilsson R, et al. (2013) Employing 454 amplicon pyrosequencing to reveal intragenomic divergence in the internal transcribed spacer rDNA region in fungi. Ecology and Evolution 3: 1751-1764.

Lubock NB, Zhang D, Sidore AM, Church GM, Kosuri S (2017) A systematic comparison of error correction enzymes by nextgeneration sequencing. Nucleic Acids Research 45: 9206-9217.

Maleszka R, Clark-Walker G (1993) Yeasts have a four-fold variation in ribosomal DNA copy number. Yeast 9: 53-58.

Medinger R, Nolte V, Pandey RV, et al. (2010) Diversity in a hidden world: potential and limitation of next-generation sequencing for surveys of molecular diversity of eukaryotic microorganisms. Molecular Ecology 19: 32-40.

Naidoo K, Steenkamp ET, Coetzee MP, Wingfield MJ, Wingfield BD (2013) Concerted evolution in the ribosomal RNA cistron. PLOS One 8: e59355.

Nei M, Rooney AP (2005) Concerted and birth-and-death evolution of multigene families. Annual Review of Genetics 39: 121.

Schoch CL, Robbertse B, Robert V, et al. (2014) Finding needles in haystacks: linking scientific names, reference specimens and molecular data for Fungi. Database- the Journal of Biological Database and Curation 2014.

Schoch CL, Seifert KA, Huhndorf S, et al. (2012) Nuclear ribosomal internal transcribed spacer (ITS) region as a universal DNA barcode marker for Fungi. Proc Natl Acad Sci U S A 109: 6241-6246.

Simon UK, Weiß M (2008) Intragenomic variation of fungal ribosomal genes is higher than previously thought. Molecular Biology and Evolution 25: 2251-2254.

Stielow J, Lévesque C, Seifert K, et al. (2015) One fungus, which genes? Development and assessment of universal primers for potential secondary fungal DNA barcodes. Persoonia-Molecular Phylogeny and Evolution of Fungi 35: 242. 
Susca A, Perrone G, Cozzi G, et al. (2013) Multilocus sequence analysis of Aspergillus Sect. Nigri in dried vine fruits of worldwide origin. International Journal of Food Microbiology 165: 163-168.

Vu D, Groenewald M, Szöke S, et al. (2016) DNA barcoding analysis of more than 9000 yeast isolates contributes to quantitative thresholds for yeast species and genera delimitation. Studies in Mycology 85: 91-105.

Vydryakova GA, Van DT, Shoukouhi P, Psurtseva NV, Bissett J (2012) Intergenomic and intragenomic ITS sequence heterogeneity in Neonothopanus nambi (Agaricales) from Vietnam. Mycology 3: 89-99.

Wang W, Ma L, Becher $\mathrm{H}$, et al. (2015) Astonishing 35S rDNA diversity in the gymnosperm species Cycas revoluta Thunb. Chromosoma: 1-17.
West C, James SA, Davey RP, Dicks J, Roberts IN (2014) Ribosomal DNA sequence heterogeneity reflects intraspecies phylogenies and predicts genome structure in two contrasting yeast species. Systematic Biology 63: 543-554.

Woo PC, Leung S-Y, To KK, et al. (2010) Internal transcribed spacer region sequence heterogeneity in Rhizopus microsporus: implications for molecular diagnosis in clinical microbiology laboratories. Journal of Clinical Microbiology 48: 208-214.

Yurkov A, Guerreiro MA, Sharma L, Carvalho C, Fonseca A (2015) Multigene assessment of the species boundaries and sexual status of the basidiomycetous yeasts Cryptococcus flavescens and C. terrestris (Tremellales). PloS One 10: e0120400. 